\title{
Intranuclear Distribution of Chiasmata in Normal Diploids and Interchange Heterozygotes of Pisum sativum L.
}

\author{
M. C. Joshi ${ }^{1}$ and K.P.S. Chauhan ${ }^{2}$ \\ Department of Plant Breeding, U. P. Agricultural \\ University, Pantnagar, India
}

Received September 17, 1971

\section{Introduction}

Morgan et al. (1933) reported that a decrease in the frequency of genetic crossing over in two of the chromosomes resulted in an increased crossing over in the third chromosome of Drosophila. Similar interchromosomal effects with respect to chiasma formation were reported by Mather (1936), Elliot (1958) and Rowlands (1958) in several organisms. The precise significance of these observations, however, remained obscure as these effects were found to be highly variable at the inter as well as intraspecies level. Later it was found in Delphinium ajacis that the chiasma formation among the different bivalents of a cell was negatively correlated in all the normal plants studied (Jain and Maherchandani 1961, Basak and Jain 1963, 1964). These authors indicated that this consistency in showing negatively correlated distribution of chiasmata was due to the highly asymmetrical karyotype possessed by this species. A similar study conducted in Chrysanthemum carinatum, which possesses a symmetrical karyotype showed that chiasmata were formed in an independent or uncorrelated manner among the different bivalents of a cell (Khamankar and Jain 1965). Thus, it appeared from the work of these authors that karyotype symmetry is an important factor in determining the manner in which chiasmata are distributed among the different bivalents of a cell.

The present investigation was undertaken to study whether Pisum sativum possessing a symmetrical karyotype also shows the absence of negatively correlated chiasma formation, as was observed in Chrysanthemum carinatum. The analysis was further extended to a number of interchange heterozygotes of this species in order to study the effect of interchange heterozygosity on the intranuclear distribution of chiasmata.

\section{Material and methods}

The study was conducted in the following two types of plants of Pisum sativum $(2 \mathrm{n}=14)$, variety $\mathrm{T}, 163$ :

\section{Diploid normal plants}

1 Graduate student, Department of Plant Breeding.

2 Assistant Professor of Plant Breeding.

U.P.A.U. Experiment Station Journal Publication Series No. 333. 
The karyotype of the diploid normal plants of this variety is largely symmetrical, according to the classification of Stebbins (1958). Due to their similarity in size, it is not possible to identify the individual chromosomes or to classify them into different groups during meiotic metaphase I stage.

\section{Interchange heterozygotes}

In order to recognize the particular chromosome pairs for observing the effect of an increase or decrease in their chiasma frequency on the other chromosome pairs, it was considered necessary to artificially induce size differences in the different chromosomes. This was achieved by irradiating the dry seeds with a gamma ray dose of 20,000 ' $r$ '. The interchange heterozygotes, screened from a large irradiated population, were found to have two groups of chromosomes: i) Five normal bivalents and ii) An interchange multiple of four chromosomes.

Meiotic studies: Young flower buds were fixed in acetic alcohol (1 part glacial acetic acid saturated with iron acetate +3 parts absolute alcohol). Anthers were squashed in 2 per cent acetocarmine solution. Scoring of chiasmata was done at meiotic metaphase I stage in well spread, randomly selected pollen mother cells from temporary preparations. Twenty five cells from each plant were studied.

Statistical analysis: The seven bivalents of Pisum sativum can not be regularly distinguished from one another because of symmetrical karyotype, and so the distribution of chiasma frequencies for any specific bivalent can not be found. The existence of a correlation for chiasma formation among the various bivalents of the same nucleus, can be tested by the method of analysis of variance given by Mather (1936, 1951), which makes it possible to partition the total variation affecting chiasma frequency into two components-a) between nuclei, b) within nuclei. The form of the analysis of variance is given below:

\section{Analysis of variance}

$\begin{array}{lc}\text { Sources of variation } & \text { Degrees of freedom } \\ \text { Between nuclei } & n-1 \\ \text { Within nuclei } & (\mathrm{m}-1) \mathrm{n} \\ \text { Total } & \mathrm{mn}-1 \\ \mathrm{~m}=\text { number of bivalents in a nucleus, } \\ \mathrm{n}=\text { number of nuclei studied in a plant. }\end{array}$

The significance of the difference between the two items namely, within nuclei and between nuclei variation can be tested by calculating the variance ratio. The significant difference between these two mean squares indicates the presence of correlation for chiasma formation among the seven bivalents. If the between nuclei variance is smaller than the within nuclei variance, the correlation is negative among the bivalents of a nucleus and if the between nuclei variance is larger than within nuclei variance, the correlation is positive among the seven bivalents of a nucleus.

The correlation for chiasma formation between two groups of chromosomes in the pollen mother cells of interchange heterozygotes was studied following the method of analysis of variance given by Mather (1936) and Elliot (1958). The analysis of variance makes it possible to divide the total variation affecting chiasma 
formation into two components, viz., between nuclei (inter-nuclear variance), between groups of chromosomes and between groups of chromosomes within nuclei (inherent variance). The study of distribution is based on the consideration that the relative magnitude of the internuclear and inherent variance indicates the direction and the extent of interchromosomal correlation in chiasma distribution. The internuclear variance measures the variation in the total number of chiasmata between the pollen mother cells in a plant, whereas, the inherent variance measures the variation in the distribution of chiasma between the distinct groups of chromosomes within nuclei. If the internuclear and inherent variance are found to be equal in their magnitude, the correlation for chiasma formation between the different groups of chromosomes does not exist. If the inherent variance exceeds the internuclear variance, it can be concluded that the different groups of chromosomes in the cell show a negatively correlated chiasma formation. A positive correlation between the groups is indicated, if the internuclear variance exceeds the inherent variance.

Analysis of variance

$\begin{array}{lc}\text { Sources of variation } & \text { Degrees of freedom } \\ \text { Between nuclei } & n-1 \\ \text { Between groups of chromosomes } & 1 \\ \begin{array}{l}\text { Internuclear (inherent; between } \\ \text { groups, within nuclei) }\end{array} & 1(\mathrm{n}-1) \\ \text { Total } & 2 \mathrm{n}-1 \\ \mathrm{n}=\text { number of nuclei studied in a plant. }\end{array}$

\section{Experimental results}

1. Intranuclear distribution of chiasmata in normal diploid plants

Observations on chiasma frequency were taken from twentyfive pollen mother cells each of seventeen plants of this group. The mean number of chiasmata per cell in these plants is given in Table 1. The distribution of chiasmata among the seven pairs of chromosomes was studied in each plant by estimating the inter and intranuclear variances for chiasma frequency, which are presented in Table 1.

It is seen from the table that in fourteen plants out of seventeen, the variance ratios are statistically insignificant. This indicates that there is no correlation for chiasma formation among the seven bivalents in these fourteen plants. The inter and intranuclear variance ratios are significant in only three plants. In these three plants the intranuclear variance is higher than internuclear, indicating a negative correlation for chiasma formation among the seven bivalents.

\section{Intranuclear distribution of chiasmata in interchange heterozygotes}

The plants of this group were isolated after a cytological screening of a large irradiated population. Eight of these plants were found to have a reciprocal interchange of chromosome segments between two of the chromosome pairs. In such plants, an interchange multiple of four chromosomes was regularly formed. The distribution of chiasmata in the pollen mother cells has, therefore, been studied 
Table 1. Internuclear and intranuclear variances in normal diploid plants

\begin{tabular}{c|c|c|c|c}
\hline Plant no. & $\begin{array}{c}\text { Mean no. of } \\
\text { chiasmata } \\
\text { per cell }\end{array}$ & $\begin{array}{c}\text { Internuclear } \\
\text { variance }\end{array}$ & $\begin{array}{c}\text { Intranuclear } \\
\text { variance }\end{array}$ & $\begin{array}{c}\text { Variance } \\
\text { ratio }\end{array}$ \\
\hline 1 & 16.15 & 0.1695 & 0.2381 & 1.4047 \\
2 & 17.96 & 0.1131 & 0.3127 & $2.7648^{* *}$ \\
3 & 16.45 & 0.2176 & 0.3309 & 1.5213 \\
4 & 16.20 & 0.0842 & 0.2547 & $3.0249^{* *}$ \\
5 & 16.28 & 0.2323 & 0.2857 & 1.2298 \\
6 & 16.35 & 0.3349 & 0.2904 & 1.1532 \\
7 & 17.50 & 0.1896 & 0.2833 & 1.4941 \\
8 & 15.85 & 0.1244 & 0.1571 & 1.2628 \\
9 & 15.16 & 0.1714 & 0.1952 & 1.1388 \\
10 & 16.50 & 0.2932 & 0.3381 & 1.1531 \\
11 & 16.35 & 0.2447 & 0.2547 & 1.0408 \\
12 & 16.00 & 0.1428 & 0.2152 & 1.5070 \\
13 & 17.26 & 0.1274 & 0.3031 & $2.3791^{* *}$ \\
14 & 17.16 & 0.2342 & 0.3581 & 1.5290 \\
15 & 14.84 & 0.2581 & 0.2932 & 1.1359 \\
16 & 16.40 & 0.3368 & 0.4428 & 1.3147 \\
17 & 16.10 & 0.3142 & 0.2952 & 1.0643 \\
\hline
\end{tabular}

** Significant at $1 \%$ level of significance.

between the interchange multiple of four chromosomes constituting one group and the five bivalents constituting the other. Observations on chiasma frequency were taken in these eight plants. The mean number of chiasmata per cell is given in Table 2. The distribution of chiasmata between the interchange multiple and the five bivalents was studied in each plant by estimating the inter and intranuclear variances, which are presented in Table 2.

It is seen from the table that in five of the eight plants, variance ratios are insignificant. Thus, these plants do not show any correlation with regard to

Table 2. Internuclear and intranuclear variances in the interchange heterozygotes

\begin{tabular}{c|c|c|c|c}
\hline \hline Plant no. & $\begin{array}{c}\text { Mean no. of } \\
\text { chiasmata } \\
\text { per cell }\end{array}$ & $\begin{array}{c}\text { Internuclear } \\
\text { variance }\end{array}$ & $\begin{array}{c}\text { Intranuclear } \\
\text { variance }\end{array}$ & $\begin{array}{c}\text { Variance } \\
\text { ratio }\end{array}$ \\
\hline 1 & 15.44 & 0.1914 & 0.2572 & 1.3437 \\
2 & 15.85 & 0.1845 & 0.1359 & 1.3576 \\
3 & 15.97 & 0.2318 & 0.0927 & $2.5005^{*}$ \\
4 & 17.56 & 0.1742 & 0.1317 & 1.3227 \\
5 & 15.80 & 0.2737 & 0.0865 & $3.1641^{* *}$ \\
6 & 15.60 & 0.2314 & 0.0738 & $3.1355^{* *}$ \\
7 & 15.20 & 0.1293 & 0.1727 & 1.3356 \\
8 & 15.20 & 0.1309 & 0.1237 & 1.0582 \\
\hline
\end{tabular}

* Significant at $5 \%$ level of significance.

** Significance at $1 \%$ level of significance. 
chiasma formation between two groups of chromosomes. In rest of the three plants the variance ratios are statistically significant. The internuclear variance exceeds the intranuclear variance in these three plants. This indicates the existence of a positive correlation for chiasma formation between the interchange multiple and five normal bivalents.
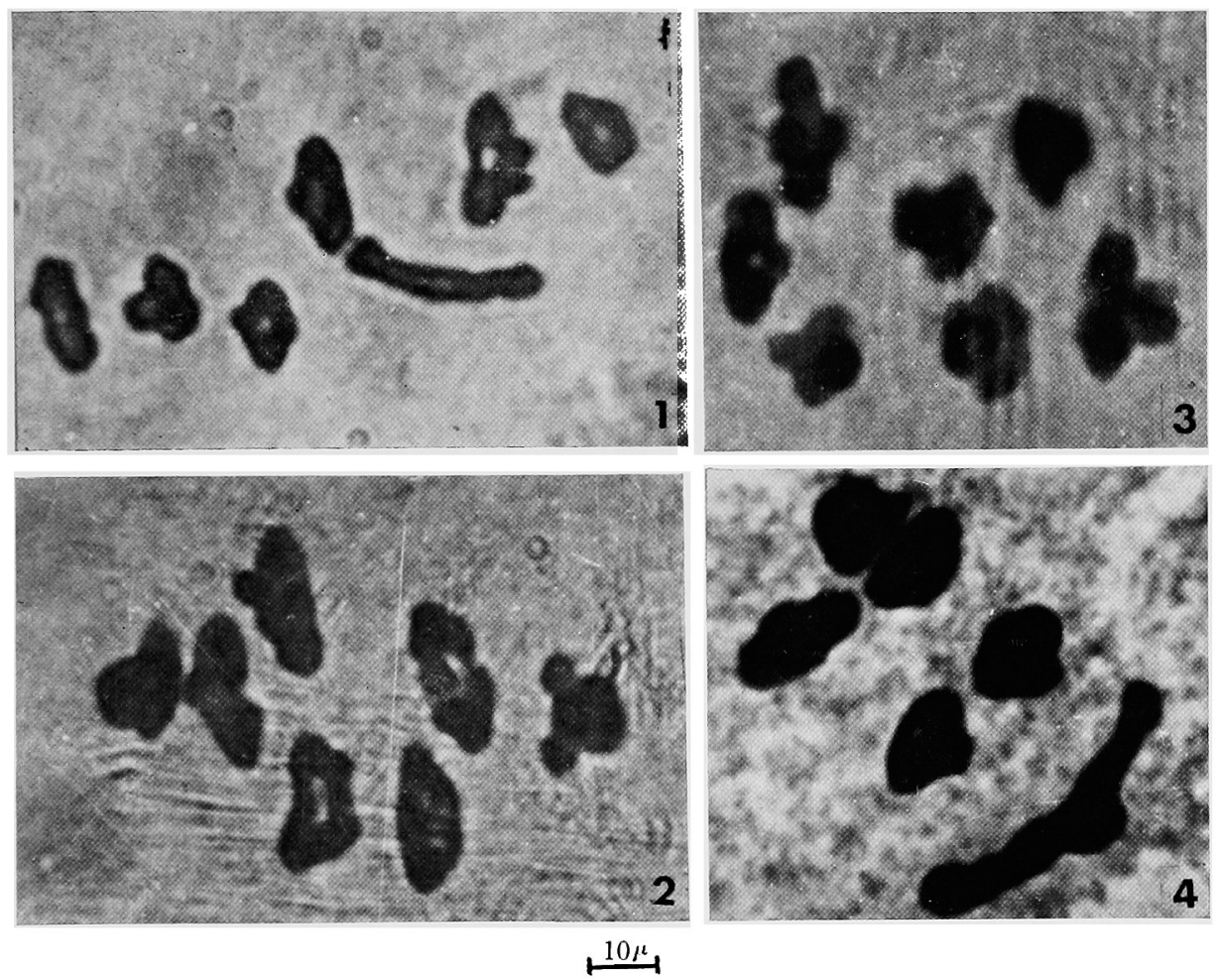

Figs. 1-4. PMCs at metaphase I stage showing variations in the intranulcear distribution of chiasmata. 1, seven bivalents with a total of 14 chiasmata $(3+2+2+2+2+2+1)$. 2, seven bivalents with a total of 15 chiasmata $(3+2+2+2+2+2+2)$. 3 , seven bivalents with a total of 17 chiasmata $(3+3+3+3+2+2+1)$. 4 , one interchange multiple and 5 normal bivalents with a total of 4 and 12 chiasmata $(3+3+2+2+2)$ respectively.

\section{Discussion}

The present observations in Pisum sativum support the findings of Khamankar and Jain (1965) in Chrysanthemum carinatum that species having a highly symmetrical karyotype tend to show absence of negative type of interchromosome correlations for chiasma formation. It is logical here to analyse the results obtained in Pisum sativum in the light of the hypothesis proposed by Basak and Jain (1963). According to this hypothesis the total chiasma frequency of a cell can be partitioned into two fractions-an autonomous fraction and a correlated fraction. It was suggested that each chromosome pair in a cell is capable of forming a certain minimum number of chiasmata independently of the other pairs. This combined 
minimum capacity of all the pairs to form the chiasmata constitutes the autonomous fraction. The correlated fraction on the other hand, is one which is left after the autonomous fraction is accounted. Therefore, the cells having a lower chiasma frequency will have most of the chiasmata formed autonomously and only a few or none formed in a correlated manner. Other cells in the same plant having a higher chiasma frequency may have a larger correlated fraction for which the different bivalents may show competition, since the autonomous fraction, as the hypothesis assumes, remains same in a plant. The hypothesis, thus, suggested that the relative magnitude of the two fractions of chiasma frequency in an organism plays an important role in determining the type of interchromosomal correlationsa lower autonomous fraction and a higher correlated fraction resulting in negative type of correlation and vice versa.

\section{8
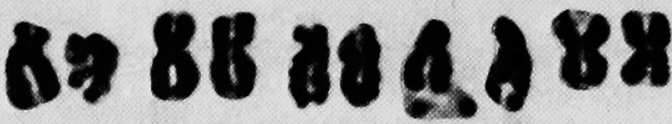 \\ A \\ B \\ C \\ D \\ E \\ $\mathbf{F}$ \\ 6}

Fig. 5. Showing karyotype of Pisum sativum var. T 163.

Table 3. Classification of different chromosomes on the basis of their morphology

\begin{tabular}{c|c|c|c}
\hline \hline $\begin{array}{c}\text { Chromosome } \\
\text { no. }\end{array}$ & $\begin{array}{c}\text { Metaphase length } \\
\text { in } \mu\end{array}$ & Arm ratio & Position of centromere \\
\hline A & 7.0 & $1: 2.2$ & SM \\
B & 6.6 & $1: 2.0$ & SM \\
C & 5.8 & $1: 3.2$ & ST \\
D & 5.4 & $1: 1.6$ & SM \\
E & 5.4 & $1: 3.0$ & ST \\
F & 5.2 & $1: 2.2$ & SM \\
G & 4.7 & $1: 1.0$ & M \\
\hline
\end{tabular}

$\mathrm{SM}=$ Submedian; $\mathrm{ST}=$ Subterminal; $\mathrm{M}=$ Median .

A study of the karyotype of Pisum sativum, var. T 163 is necessary for understanding the probable manner-autonomous or correlated-in which chiasmata would be formed in the different chromosomes of a cell. It is seen from Fig. 5 and Table 3 that all the seven chromosomes of Pisum sativum are nearly equal in size (range $4.7 \mu-7.0 \mu$ ). As given in Table 3, chromosomes marked A, B, D and $F$ possess a submedian centromere. Each of these chromosomes is expected to form a minimum of one chiasma in each of it's arms, independently of chiasma formation in other chromosomes. The smallest chromosome $\mathrm{G}$, which possesses a median centromere would likewise form at least single chiasma in each of it's arms, since chiasma interference as a rule does not work across the centromere. However, chromosomes $\mathrm{C}$ and $\mathrm{F}$ are expected to form a single chiasma in the long arm only. Due to it's very small size, the short arm of these chromosomes may not be able 
to form a chiasma. Formation of a second chiasma in the long arm of these chromosomes would depend upon the extent of chiasma interference in that arm. Similarly, the long arm of other chromosomes will form a second chiasma depending upon the strength of chiasma interference in that arm. It is this fraction of chiasma frequency, after the autonomous or independent fraction is accounted, for which the different chromosomes may show competition. In case of a species like Pisum sativum, which has most of its chromosomes having a median or submedian centromere, it is expected, therefore, that a larger proportion of total chiasma frequency of a cell would be formed in an independent or autonomous way and a fewer chiasmata would be formed in a correlated way.

Besides chromosome morphology, genotype is another important factor which may affect the relative magnitude of the two fractions of chiasma frequency. It is known that the different plants of the same species may show differences in the extent of chiasma interference (Haldane 1931). The plants showing a higher chiasma interference are expected to show a lower autonomous fraction and a higher correlated fraction of chiasma frequency. The three plants show negative correlation for chiasma formation. A possible explanation for this may be that these plants have a genetically controlled higher chiasma interference than others.

In order to further test the applicability of the hypothesis proposed by Basak and Jain (1963), another group of plants-interchange heterozygotes, were used. In these plants it was possible to get a better estimate of the inherent variance (internuclear variance) by eliminating from it the variance due to grouping of chromosomes into a quadrivalent and normal bivalents. It is seen from the results that five plants out of eight show absence of any correlation for chiasma formation. These findings support the observations of Basak and Jain (1964) in Delphinium ajacis, where plants with one interchange complex, two interchange complexes, as well as an absence of interchange heterozygosity consistently showed negatively correlated distribution of chiasmata. Khamankar and Jain (1965) also observed no effect of interchange heterozygosity on chiasma distribution in Chrysanthemum carinatum. The three plants showing significant positive correlation indicate a decline in the intranuclear variance to a great extent, which is again an indication of the absence of negative type of correlation. The significant positive correlation implies that with an increase in the chiasma frequency in the interchange multiple, there is a corresponding increase in the chiasma frequency of the normal bivalents also. Significant positive correlations have been reported earlier sporadically and have been ascribed to environmental influences (Mather 1936).

Observations in Pisum sativum, thus, indicate that a highly symmetrical karyotype would tend to favour absence of any correlation for chiasma formation, as was proposed by Khamankar and Jain from their findings in Chyrsanthemum carinatum.

\section{Summary}

Studies on intranuclear distribution of chiasmata were carried out in two types of plants of Pisum sativum, var. T 163-1) normal diploids and 2) interchange hetero- 
zygotes. In most of the plants the chiasmata were found to be distributed in an uncorrelated manner among the different bivalents of a cell. This was ascribed to the highly symmetrical karyotype possessed by Pisum sativum.

\section{Acknowledgements}

The authors are grateful to Dr. K. G. Gollakota, Dean, College of Post-Graduate Studies, Dr. N. K. Anant Rao, Dean, College of Agriculture; Dr. Maharaj Singh, Additional Director, Experiment Station and Dr. V. L. Asnani, Professor and Head, Department of Plant Breeding for providing the necessary facilities for conducting this work.

\section{References}

Basak, S. L. and Jain, H. K. 1963. Autonomous and interrelated formation of chiasmata in Delphinium chromosomes. Chromosoma 13: 577-87.

- and - 1964. The interchromosome distribution of chiasmata in interchange heterozygotes of Delphinium. Heredity 19: 53-61.

Elliot, C. D. 1958. Environmental effects on the distribution of chiasmata among nuclei and bivalents and correlation between bivalents. Heredity 12: 429-39.

Haldane, J. B. S. 1931. The cytological basis of genetical interference. Cytologia 3: 54-65.

Jain, H. K. and Basak, S. L. 1963. Genetic interpretation of chiasmata in Delphinium. Genetics 48: $329-339$.

- and - 1965. Experimental modification of chiasma distribution in Delphinium. Indian Journal of Genetics and Plant Breeding 25: 14-23.

- and Maherchandani, N. 1961. The control of intranuclear distribution of chiasmata in Delphinium. Heredity 16: 383-92.

Khamankar, Y. G. and Jain, H. K. 1965. Karyotype and the control of interchromosome distribution of chiasmata. Indian Journal of Genetics and Plant Breeding 25: 353-359.

Mather, K. 1936. Competition between bivalents during chiasma formation. Proc. Roy. Soc. London B 120: 208-227.

- 1951. Statistical Analysis in Biology. 4th ed., Methuen and Co., Ltd. London.

- and Lamm, R. 1935. The negative correlation of chiasma frequencies. Hereditas 20: 65-70.

Morgan, T. H., Bridges, C. B. and Schultz, J. 1933. Constitution of the germinal material in relation to heredity. Carne. Inst. Year Book 32: 298-302.

Rowlands, D. G. 1958. The control of chiasma frequency in Vicia faba L. Chromosoma 9: 176184.

Stebbins, G. L. 1958. Longevity, habitat and release of genetic variability in higher plants. Cold Spring Harbor Symposia in Quant. Biol. 16: 175-197. 